Trauma Berufskrankh 2006 • 8[Suppl 2]: S150-S154

DOI 10.1007/s10039-006-1156-5

Online publiziert: 28. Juni 2006

(c) Springer Medizin Verlag 2006
D. Altmann

Franziskus-Krankenhaus, Linz, Rhein

\section{Operativer Standard bei frischen}

Kreuzbandverletzungen

\author{
Healing-response-Technik - eine \\ moderne Alternative?
}

Die Kreuzbänder sind die zentralen Stabilisatoren des Knies. Ihr Verlust bewirkt eine Instabilität und Funktionsstörung des Kniegelenks, was in vielen Fällen zu Meniskus- und Knorpelschädigungen führt.

Der wichtigen Rolle der Kreuzbänder in der Funktion des Kniegelenks wurde schon im 19. Jahrhundert operativ Rechnung getragen. So wurden schon 1895 vordere Kreuzbänder genäht (Mayo Robson, Leeds 1895) oder mittels Seidenfäden augmentiert (Lang, 1903). Durch die Entwicklung der Arthroskopie in den 197oer Jahren und die rasanten Fortschritte in der Instrumententechnik ist die Kreuzbandplastik zu einer Standardoperation geworden.

Häufigste Unfallursache für Kreuzbandverletzungen ist ein Trauma

- mit $64 \%$ beim Fußballsport,

- mit 23\% beim Skifahren und

- mit 9,2\% während der Arbeit,

wobei das vordere Kreuzband (VKB) deutlich häufiger betroffen ist (in etwa $85 \%$ der Fälle). Die Kreuzbandruptur kann isoliert auftreten oder als kombinierte Verletzung mit schweren weiteren Band-, Meniskus- oder Knorpelverletzungen. Immer noch werden diese schwer wiegenden Verletzungen übersehen oder zu spät therapiert.

Insbesondere unter dem Aspekt einer neuen operativen Technik, der Healingresponse-Therapie, ist es erforderlich, die
Verletzung frühzeitig zu diagnostizieren und zu therapieren.

Im Vergleich zur Verletzung des vorderen Kreuzbands stellt die Ruptur des hinteren Kreuzbands eine noch größere Herausforderung an den behandelnden Chirurgen dar. Für die Behandlung der akuten Verletzung stehen abhängig vom Anspruch des Patienten und den Begleitverletzungen differenzierte Therapiekonzepte zur Verfügung

\section{Vordere Kreuzbandruptur}

\section{Klinik und Diagnostik}

Bei der akuten vorderen Kreuzbandverletzung kann die klinische Diagnostik schwierig sein, da der Patient primär, bei fehlenden Begleitverletzungen, keine großen Schmerzen angibt. Des Weiteren behindert der auftretende Hämarthros die Diagnostik.

Der Lachman-Test hat die größte Aussagekraft, ist aber nur selten stark ausgeprägt. Beim Vorliegen eines Hämarthros kann die Indikation zur sofortigen Arthroskopie gestellt werden, da bei einem adäquaten Trauma in über $\mathbf{9 2} \%$ der Fälle ein operativ zu sanierender Kniebinnenschaden vorliegt (eigene nicht veröffentlichte Studie, 1986, bei 200 arthroskopisch kontrollierte Kniegelenken).

Nach Ausschluss einer knöchernen Verletzung durch Standardröntgenbilder kann eine Magnetresonanztomographie im Akutstadium in guter Qualität Kniebinnenschäden (Bone bruises, Impressionsfrakturen, Knorpelflakes, Art und Lokalisation von Kapsel-Band-Verletzungen sowie Meniskusläsionen) abbilden.

\section{Operation}

Nach wie vor ist der ideale Versorgungszeitpunkt einer frischen VKB-Ruptur wegen der möglichen Arthrofibroseentwicklung umstritten. Entscheidend für diese ist nicht der Operationszeitpunkt, sondern der Reizzustand des Knies. Es scheinen jedoch keine Arthrofibroseprobleme zu entstehen, wenn innerhalb der ersten 5 Tage operiert wird und keine wesentliche Funktionsstörung vorliegt [6]. Unser

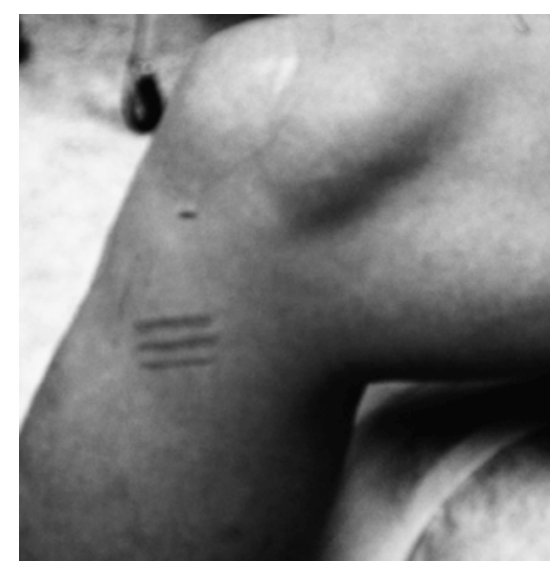

Abb. 1 \ Pes-anserinus-Sehnen 
neues Vorgehen, die Healing-responseTechnik, zeigte keine Arthrofibroseentwicklung. Deshalb sollten sie oder die Kreuzbandplastik zur akuten Versorgung der frischen Kreuzbandruptur angestrebt werden.

Ziel einer operativen Rekonstruktion des rupturierten VKB ist die Wiederherstellung eines möglichst physiologischen Transplantatverhaltens und damit eines annähernd normalen Roll-Gleit-Mechanismus. Dies ist bei der Healing-responseTechnik wegen des Reattachments des Kreuzbands an anatomischer Stelle gegeben. Beim Transplantat ist die richtige Positionierung der Bohrkanäle für ein gutes Ergebnis nach vorderer Kreuzbandplastik entscheidend. Fehllagen führen

- zur Bewegungseinschränkung,

- zum Transplantatversagen oder

- zur bleibenden Instabilität.

Transplantatwahl. Die derzeit verwendeten Transplantate sind autologe Gewebe:

- Patellarsehne,

- Hamstring-Sehnen als 4fach-Transplantat sowie

- Quadrizepssehne.

Seltener wird ein Leichentransplantat eingesetzt. Die einzelnen Transplantate weisen spezifische Unterschiede bezüglich der biomechanischen Eigenschaften, der Primärfixation und der Einheilung im Knochenkanal auf [11]. Bei der Transplantatauswahl müssen die unterschiedlichen anatomischen und funktionellen Anforderungen berücksichtigt werden.

Transplantatverankerung. Die ossäre Transplantatintegration unterliegt spezifischen biomechanischen und biologischen Rahmenbedingungen und ist bei den Hamstring-Sehnen nach etwa 8 Wochen abgeschlossen. Eine anatomische Verankerung, möglichst nahe dem Originalkreuzbandansatz, muss angestrebt werden.

Die Verwendung von Hamstring-SehnenTransplantaten als 4 fach-Transplantat hat zunehmend an Stellenwert gewonnen [1]. Der wesentliche Vorteil dieser Sehnen gegenüber dem mittleren Patellarsehnendrittel liegt in einer erheblich reduzierten Transplantatentnahmemorbidität, geringeren vorderen Knieschmerzen (ante-

Trauma Berufskrankh 2006 · 8[Suppl 2]: S150-S154 DOI 10.1007/s10039-006-1156-5

(c) Springer Medizin Verlag 2006

\section{Altmann \\ Operativer Standard bei frischen Kreuzbandverletzungen. Healing-response-Technik - eine moderne Alternative?}

\section{Zusammenfassung}

Die Kreuzbänder sind die zentralen Stabilisatoren des Knies. Ihr Verlust bewirkt eine Instabilität und Funktionsstörung des Kniegelenks, was in vielen Fällen zu Meniskus- und Knorpelschädigungen führt. Durch die Etablierung der Arthroskopie in den 1970er Jahren und die rasante Entwicklung der Instrumententechnik ist die Kreuzbandplastik zu einer Standardoperation geworden. Zur Behandlung der Ruptur des vorderen Kreuzbands (VKB) werden die klinische und bildgebende Diagnostik sowie die operativen Standards hinsichtlich Zeitpunkt, Implantate (Transplantatwahl), Transplantatpositionierung und Fixation aufgezeigt. An einem Gesamtkollektiv von 120 Patienten wird eine neue Methode der Versorgung frischer proximaler Kreuzbandrupturen, die Healing-response-Technik, vorgestellt. Ihre arthroskopisch kontrollierten Ergebnisse bei 110 Patienten werden dargestellt. Die Ruptur des hinteren Kreuzbands stellt eine noch größere Herausforderung an den behandelnden Chirurgen dar als die Versorgung des VKB. Die Therapie der akuten Verletzung wird kontrovers diskutiert.

\section{Schlüsselwörter}

Kreuzband $\cdot$ Healing-response-Technik $\cdot$ Semitendinosussehne Anatomische Fixation . Akutes Trauma

\section{Standards in operative treatment of acute trauma of the cruciate ligaments. Healing response technique - a new alternative?}

\section{Abstract}

The cruciate ligaments are the main stabilizers of the knee. Their loss causes instability and malfunction of the knee joint, leading to severe meniscus tears and cartilage lesions in many cases. The development of arthroscopic surgery in the 1970s and the fast pace of advances in instrument technology have combined to make $\mathrm{ACL}$ reconstruction a standard procedure. Treatment of a tear of the ACL must be preceded by both clinical examination and diagnostic imaging; the standards in terms of timing, implants, transplant placement and fixation are highlighted. A new method for the treatment of acute
$\mathrm{ACL}$ tears, the healing response technique, is introduced following its first use in 120 patients; the results achieved have been monitored by arthroscopy in 110 of the patients and are also presented. Rupture of the PCL is an even greater challenge to the knee surgeon. The different opinions on its management are discussed.

\section{Keywords}

Cruciate ligaments $\cdot$ Healing response $\cdot$ Semitendinosus - Double bundle technique . Acute trauma 


\section{Kniegelenk}

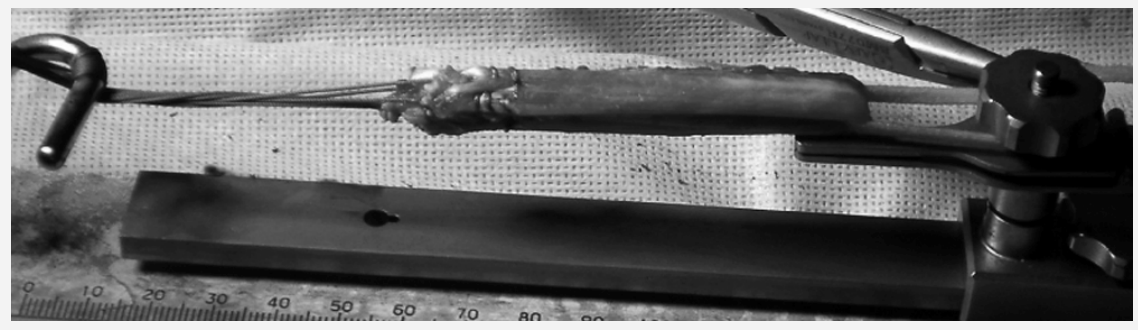

Abb. $2 \triangle$ Transplantat

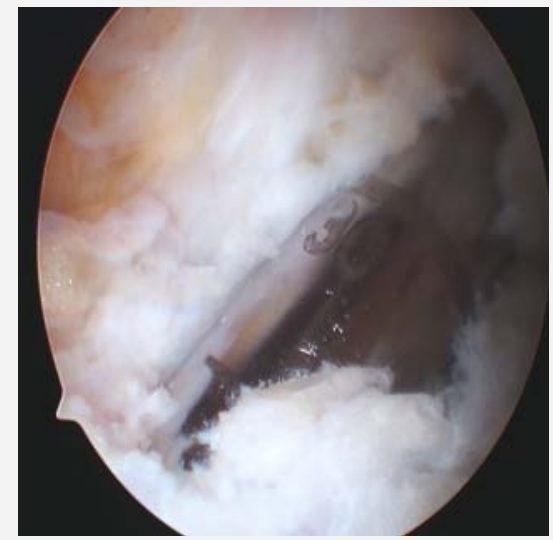

Abb. $3 \Delta$ Femoraler Kopfraumbohrer
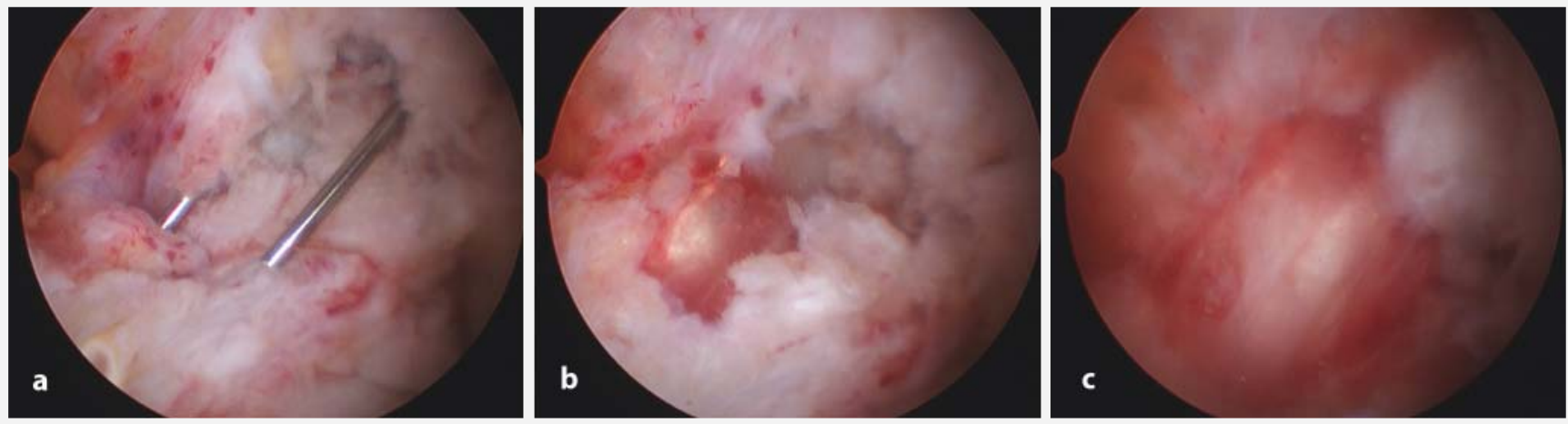

Abb. 4 \ Einziehen des Transplantats
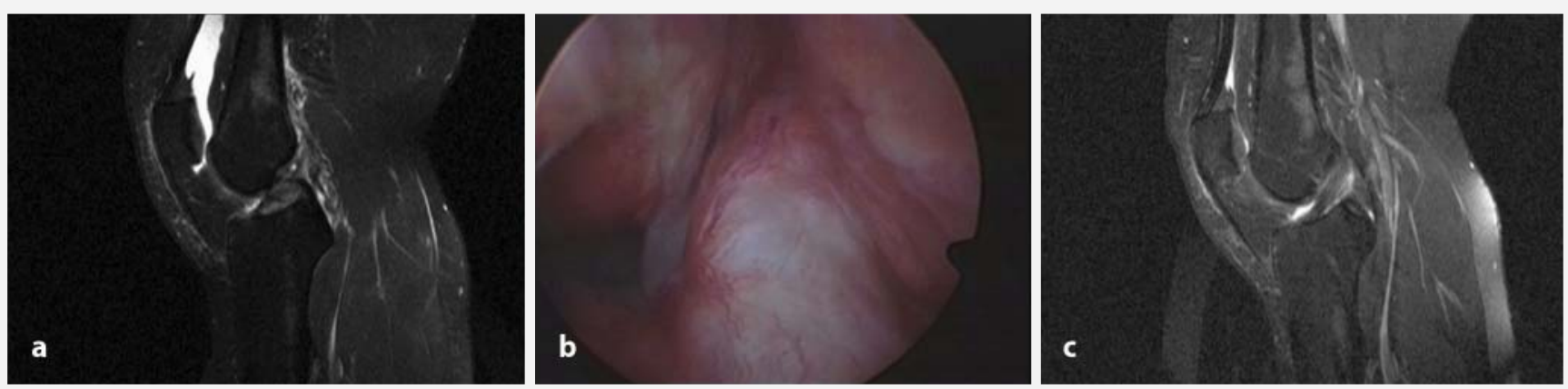

Abb. $5 \Delta$ Kreuzbandruptur, a MRT, b arthroskopisches Kontrollbild, c Kontroll-MRT

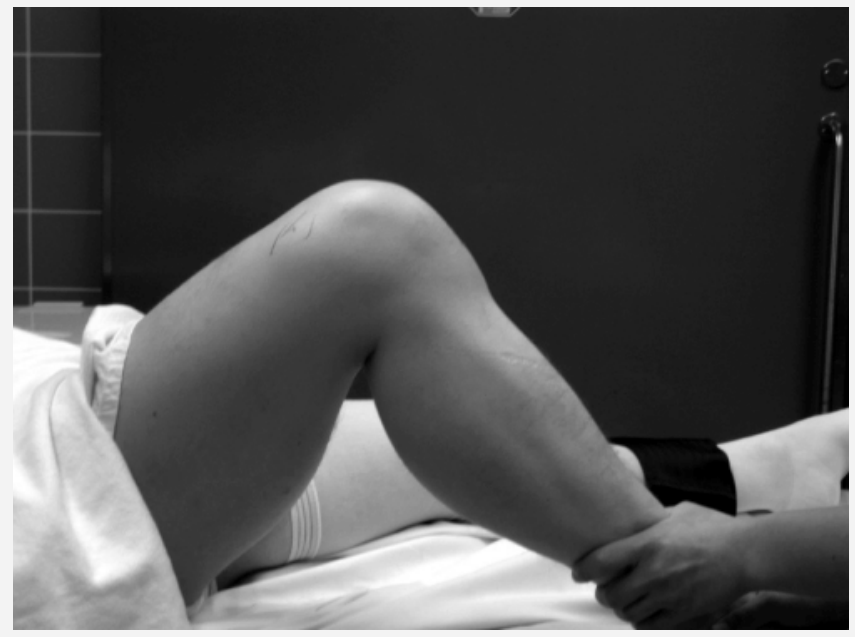

Abb. $6 \Delta$ Step-off-Test

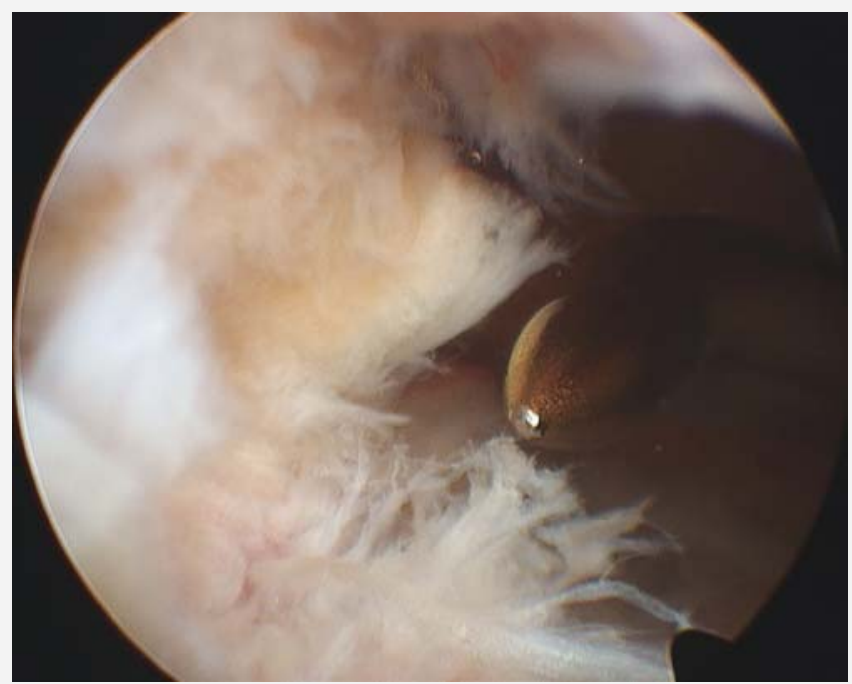

Abb. 7 A Hintere Kreuzbandruptur, posterior-medialer Zugang 


\begin{tabular}{|l|}
\hline Tab. 1 Operationsschritte TransFix- \\
Technik \\
\hline 1. Transplantatentnahme \\
\hline 2. Transplantatpräparation \\
\hline 3. Bestimmen des Tunneldurchmessers \\
\hline 4. Positionierung und Bohren der Tunnel \\
\hline 5. Einziehen des Transplantats \\
\hline 6. Femorale Fixation \\
\hline 7. Tibiale Fixation \\
\hline
\end{tabular}

Tab. 2 Ergebnisse der Healing-

response-Technik

\begin{tabular}{|ll}
\hline Operationen [Patienten] & 120 \\
\hline Arthroskopische Kontrolle [Patienten] & 110 \\
\hline Alter [Jahre] & $18-50$ \\
\hline Eingeheilt [Patienten] & 93 \\
\hline ACL auf PCL fixiert [Patienten] & 9 \\
\hline Instabile Narbe [Patienten] & 8 \\
\hline
\end{tabular}

\begin{tabular}{|llll|}
\hline Tab. 3 Stabilitätsstests & & \\
& Negativ & + & ++ \\
Lachman-Test & 100 & 11 & 9 \\
\hline Pivot-shift-Test & 112 & 8 & 0 \\
\hline
\end{tabular}

rior knee pain) und einem höheren Bewegungsausmaß. Sportler, die mit Hamstring-Sehnen versorgt wurden, weisen eine signifikant bessere Sprungkraftfunktion auf [8]. Durch die gelenknahe Aufhängung sind die typischen Probleme des Hamstring-Transplantats wie der Bungeeund der Wiper-Effekt extrem minimiert, sodass für uns diese Versorgung der goldene Standard ist.

\section{Darstellung der Operation anhand der Transfixationstechnik.}

1. Entnahme der Semitendinosussehne durch einen $2-3 \mathrm{~cm}$ großen Hautschnitt am Pes anserinus (• Abb. 1). Das freie Transplantat sollte eine Länge von $280 \mathrm{~mm}$ haben. Bei einem zu kurzem Transplantat ist die zusätzliche Entnahme der Grazilissehne erforderlich.

2. Die Sehne wird gehälftet, von Muskulatur befreit und auf einer Nahtbank präpariert ( Abb. 2).

3. Die Länge und die Dicke der resultierenden Kreuzbandplastik werden bestimmt.

4. Das tibiale Zielgerät wird tibial etwa $1 \mathrm{~cm}$ oberhalb des Pes anserinus und etwa $1,5 \mathrm{~cm}$ medial der Tuberositas tibiae sowie intraartikulär $7 \mathrm{~mm}$ vor dem hinteren Kreuzband an iso- metrischer Stelle eingesetzt, ein Bohrdraht wird vorgebohrt, die anatomische Lage überprüft und dann mit einem Bohrer überbohrt, dessen Durchmesser $1 \mathrm{~mm}$ kleiner als das ausgemessene Transplantat ist. Abschließend wird mit einem Dilatator auf die endgültige Tunnelgröße aufgedehnt.

Die femorale Zielführung wird in anatomischer "over the top"-Position bei 10.30-11.00 Uhr bzw. 14.0014.30 Uhr befestigt. Bei $90^{\circ}$ Beugung wird ein Zieldraht femoral fixiert und mit dem Kopfraumbohrer bis $35 \mathrm{~mm}$, kontrolliert an der Bohrerskala, überbohrt (- Abb. 3).

5. Der TransFix-Haken wird transtibial in den femoralen Bohrkanal eingebracht, die Haut wird inzidiert, und die Zieldrahthülse wird auf dem Knochen platziert. Nach transkutanem Durchbohren mit dem Führungsdraht nach medial, Durchziehen des flexiblen Ösendrahts und Bildung der Einzugschlaufe aus dem tibialen Bohrkanal wird das Transplantat durch kontinuierlichen Zug vollständig in den femoralen Kanal eingezogen (- Abb.4).

6. Das Bio-TransFix-Implantat wird mit dem Treiber über den Nitinolösendraht, dessen Beweglichkeit unbedingt geprüft werden muss, eingebracht und eingeschlagen. Der Nitinoldraht wird nach medial entfernt.

7. Nach maximaler Spannung des Transplantats wird dieses tibial mit einer $1 \mathrm{~mm}$ dickeren bioabsorbierbaren Schraube in $0-10^{\circ}$ Streckung fixiert (- Tab. 1).

\section{Healing-response-Technik}

Aufmerksam geworden durch die guten von Steadman et al. [10] aus Vail Colorado veröffentlichen Ergebnisse haben wir 2003 mit dieser Operationstechnik begonnen. Von Oktober 2003-März 2006 wurden 120 Patienten (67 Männer, 53 Frauen) mit diesem Verfahren operiert. Es handelte sich um frische vordere Kreuzbandabrisse am femoralen Ansatz, die innerhalb der ersten 5 Tage mittels dieser Technik versorgt wurden. Bei etwa $35 \%$ der Patienten bestanden begleitende Verlet- zungen wie Meniskus-, Knorpel- oder Seitenbandverletzungen, die in der gleichen Sitzung operiert wurden.

Bei 110 Patienten wurde nach 1012 Wochen bei freier Funktion eine Kontrollarthroskopie durchgeführt.

Technik. Nach dem Wolf-Gesetz können sich undifferenzierte Stammzellen aus dem Knochenmark in Abhängigkeit von der mechanischen Beanspruchung zu Tenozyten ausdifferenzieren. Aufgrund dieser Theorie werden nach Abschluss der diagnostischen Arthroskopie und der Versorgung der Begleitschäden mit einer speziellen Ahle bis zu 10 Perforationen an der femoralen Insertionsstelle des Kreuzbands gesetzt. Auf einen ausreichenden Blutaustritt wird geachtet. Das Kreuzband wird minutiös mit einem speziellen Instrument an die anatomische Ansatzstelle in den Blutkoagel unter gleichzeitiger Streckung des Kniegelenks reponiert. Es wird keine Gelenkdrainage benutzt. Anschließend wird das Kniegelenk in einer Schiene mit $10^{\circ}$ Beugestellung für 6 Wochen fixiert.

Die Belastung wird unter Beachtung der Begleitverletzungen definiert. Nach 6 Wochen werden die Funktion freigegeben und ein intensives Training durchgeführt.

Ergebnisse. Die arthroskopische Kontrolle bei 110 Patienten erfolgte 10-14 Wochen nach der Erstoperation und war mit den Patienten bei der Primärversorgung besprochen worden ( $\mathbf{0}$ Abb. 5).

In $85 \%$ war das Kreuzband an anatomischer Stelle eingeheilt. Die klinischen Untersuchungsergebnisse zeigten nur bei 1 Patientin eine wesentliche Funktionseinschränkung und bei 2 weiteren eine endgradiges Beugedefizit von $10^{\circ}$. Alle anderen 118 Patienten hatten ein seitengleiches Bewegungsspiel (@ Tab. 2, 3).

\section{Resümee.}

- Die Healing-response-Technik funktioniert.

- Die Healing-response-Technik ist eine sehr wirtschaftliche Alternative zur Kreuzbandplastik.

- Die Healing-response-Technik sollte auf ältere Patienten ausgedehnt werden. 
- Eine prospektive Studie sollte sich anschließen.

\section{Hinteres Kreuzband}

Eine Ruptur desselben ist eher selten, die Häufigkeitsangaben schwanken je nach Zitat zwischen 3 und $44 \%$ bei schweren Kapsel-Band-Verletzungen [2]. Insbesondere bei Ballsportlern wurde über eine relativ hohe Zahl berichtet [4].

\section{Klinik und Diagnostik}

Häufige Ursachen sind direkte Anpralltraumen (dash-board injury), auch sollte man bei Polytraumen mit Knieverletzungen an eine mögliche hintere Kreuzbandruptur denken.

Bei der frischen Verletzung des hinteren Kreuzbands zeichnet sich die Klinik im Gegensatz zur VKB-Verletzung durch deutlich größere Schmerzen und seltenere Ergussbildung aus. Ein Hämatom lässt sich oft in der Kniekehle finden, dort ist auch meist der Schmerz lokalisiert. Typische Tests sind der Hyperextensionsund der Step-off-Test (• Abb. 6) [4].

Eine knöcherne Verletzung muss dringlich ausgeschlossen werden (Standardröngten). Eine wesentliche Aussage ergibt die gehaltene Aufnahme nach Scheuba in der Auswertungstechnik von Jacobsen [3]. Die Magnetresonanztomographie kann die Diagnostik ergänzen.

\section{Operation}

Nach wie vor ist sie beim akuten hinteren Kreuzbandesriss die Ausnahme (• Abb. 7).

Bedingt durch die gute Heilungstendenz und die guten funktionellen Ergebnisse der konservativen Therapie besteht eine Indikation zum chirurgischen Eingriff in erster Linie bei komplexen Bandverletzungen mit Beteiligung des posterolateralen Komplexes oder des vorderen Kreuzbands [5].

Bei einer hinteren Schublade $>12 \mathrm{~mm}$ muss von einer kombinierten Verletzung ausgegangen werden.

Einige Autoren stellen die Indikation zur primären Operation zur Vermeidung der fixierten hinteren Schublade [7].
Operationstechniken. Die konkurrierenden Verfahren sind

- die anatomische Rekonstruktion des anterolateralen Hauptbündels in Single-incision-Technik mittels Hamstring-Sehnen [12],

- die anatomische Rekonstruktion beider Bündel sowie

- die Tibial-Inlay-Technik [7].

Die anatomische Doppelbündeltechnik mit einem Semitendinosus-Grazilissehnen-Y-Transplantat ist für uns derzeit die Methode der Wahl.

Bei 2 Patienten haben wir bei einer kombinierten Kreuzbandverletzungen eine Healing-response-Technik für das hintere Kreuzband erprobt.

Wesentlich für das postoperative Ergebnis ist eine adäquate Nachbehandlung. Lagerung in der PTS-Schiene für 6 Wochen Tag und Nacht und krankengymnastische passive Mobilisation bis $70^{\circ}$ in Bauchlage sind Inhalte derselben [12].

\section{Fazit}

Bei der akuten vorderen Kreuzbandverletzung ist die Healing-response-Technik eine beachtenswerte Alternative, deren Stellenwert zukünftig durch eine prospektive Studie erhärtet werden muss. Die Versorgung der akuten hinteren Kreuzbandverletzung ist derzeit die Domäne der konservativen Versorgung. Die Healing-response-Technik wird erprobt.

\section{Korrespondierender Autor}

\section{Dr. D. Altmann}

Franziskus-Krankenhaus

Magdalena-Dämen-Straße 20,

53545 Linz, Rhein

d.altmann@krankenhaus-linz.de

Interessenkonflikt. Es besteht kein Interessenkonflikt. Der korrespondierende Autor versichert, dass keine Verbindungen mit einer Firma, deren Produkt in dem Artikel genannt ist, oder einer Firma, die ein Konkurrenzprodukt vertreibt, bestehen. Die Präsentation des Themas ist unabhängig und die Darstellung der Inhalte produktneutral.

\section{Literatur}

1. Cony IS, Webb JM, Olingelefter AJ et al. (1999) Arthroscopic reconstruction of the anterior cruciate ligament: a comparison of patellar tendon autograft and four-strand hamstring tendon autograft. Am J Sports Med 27: 444-454
2. Fanelli GC, Edson CJ (1995) Posterior cruciate ligament injuries in trauma patients: Part II. Arthroscopy 5: 526-529

3. Jacobsen K (1976) Stress radiographical measurement of the antero posterior, medial and lateral stability of the knee joint. Acta Orthop Scand 3: 335-340

4. Jung T, Strobel M, Weiler A (2006) Diagnostik und Therapie von Verletzungen des hinteren Kreuzbandes. Unfallchirurg 1009: 41-60

5. Lobenhoffer P, Lattermann C, Krettek C et al. (1996) Rupturen des hinteren Kreuzbandes: heutiger Behandlungsstand. Unfallchirurg 99: 382-399

6. Mayr HO (2000) Arthrolyse nach vorderer Kreuzbandrekonstruktion. Arthroskopie 1/2: 54-59

7. Noyes FR, Barber-Westin S (2005) Posterior cruciate ligament replacement with a two-strand quadriceps tendon-patellar bone autograft and a tibial inlay technique. J Bone Joint Surg Am 87: 1241-1252

8. Oberthaler G, Aichner A, Marth W et al. (2003) Vorderer Kreuzbandersatz mit Semitendinosus-Grazilis-Sehne 4 fach vs. Lig. patellae vs. funktioneller Behandlung. Arthroskopie 4: 255-261

9. Stähelin AC, Südkamp NP, Weiler A (2001) Anatomic double-bundle posterior cruciate ligament reconstruction using hamstring tendons. Arthroscopy 17: 88-97

10. Steadman R, Cameron ML, Briggs KK et al. (2002) Healing-response treatment for $\mathrm{ACL}$ injuries. Orthop Technol Rev 3: 3

11. Weiler A (2000) Aktuelle Aspekte in der Verankerung von Hamstringsehnen-Transplantaten in der Kreuzbandchirurgie. Chirurg 9: 1034-1044

12. Weiler A, Jung T, Strobel M (2006) Arthroskopisch assistierte hintere Kreuzbandplastik und posterolaterale Stabilisierung mit Semitendinosus-Gracilissehnen. Unfallchirurg 109: 61-71 